\title{
Subtipo Respiratório Versus Não Respiratório no Transtorno de Pânico com Agorafobia: Avaliação com Terapia Cognitivo-Comportamental
}

\author{
Respiratory Versus Non Respiratory Subtypes of Panic Disorder \\ with Agoraphobia: Cognitive Behavioral Therapy Assessment
}

\author{
Anna Lucia Spear King*, , Alexandre Martins Valença ${ }^{b}$, José Pedro Simões Neto ${ }^{a, c}$, \\ Antonio Egidio Nardi ${ }^{a} \&$ Adriana Cardoso de Oliveira e Silva ${ }^{a, b}$ \\ ${ }^{a}$ Universidade Federal do Rio de Janeiro, Rio de Janeiro, Brasil, \\ ${ }^{b}$ Universidade Federal Fluminense, Niterói, Brasil \\ \& ' Instituto Universitário de Pesquisas do Rio de Janeiro, Rio de Janeiro, Brasil
}

\begin{abstract}
Resumo
Objetivo: verificar a resposta dos pacientes com transtorno de pânico com agorafobia à modelo proposto de terapia cognitivo-comportamental (TCC) nos dois subtipos respiratórios de transtorno de pânico: o subtipo respiratório (SR) e subtipo não respiratório (SNR) Amostra randomizada por sorteio com 50 pacientes diagnosticados segundo o Manual Diagnóstico e Estatístico dos Transtornos Mentais. A medicação: antidepressivos tricíclicos ou inibidores seletivos de recaptação da serotonina. Setenta e sete ponto seis porcento da amostra de pacientes de ambos os grupos apresentaram o SR e 22,4 \% o SNR. Os pacientes do $\mathrm{SR}$, responderam satisfatoriamente ao tratamento com técnicas da TCC, reduzindo ansiedade, sintomas respiratórios e os ataques de pânico. Os pacientes do SR melhoraram, segundo a escala de avaliação global do funcionamento, de 55,8 para 70,9 em comparação com o SNR.
\end{abstract}

Palavras-chave: Terapia cognitivo-comportamental, subtipo respiratório, pânico, ansiedade.

\begin{abstract}
The objective of the present study was to verify the response of patients with panic disorder (agoraphobia) to existing cognitive-behavior therapy models (CBT) of two respiratory subtypes of panic disorder (PD): respiratory subtype (RS) and non respiratory subtype (NRS). We randomly selected a sample of 50 patients diagnosed according to the Diagnostic and Statistical Manual of Mental Disorders. The medication used was tricycle or selective serotonin reuptake inhibitor antidepressants. Seventy-seven point six percent of the patients from both groups showed RS and 22.4\% the NRS. The RS patients responded satisfactorily to the treatment with techniques of CBT decreasing anxiety, breathing symptoms and panic attacks. According to the global functioning assessment scale, RS patients improved from 55.8 to 70.9 in comparison with NRS ones.

Keywords: Cognitive-behavior therapy, respiratory subtype, panic, anxiety.
\end{abstract}

Segundo o Manual Diagnóstico e Estatístico de Transtornos Mentais da Associação Americana de Psiquiatria (APA, 2000), o transtorno de pânico (TP) se caracteriza por ataques de ansiedade agudos, freqüentes e recorrentes que resultam em comportamentos fóbicos e inadequados.

O TP começa a se constituir a partir do momento em que o indivíduo ansioso começa a associar erroneamente as sensações corporais com algo ameaçador. A partir de uma situação de estresse ou experiência traumática, uma

"Endereço para correspondência: Rua Almirante Gomes Pereira, 8, Urca, Rio de Janeiro, RJ, Brasil 22291-170. Tel.: (21) 22954477, (21) 92191233. E-mail: annaluciaking@gmail.com cognição distorcida em relação a essas sensações se estabelece e passa a ser o padrão de funcionamento vigente no indivíduo. Mesmo em uma situação segura se a mente interpretar como insegura, o corpo reagirá como uma mensagem de perigo produzindo sintomas.

Essa relação equivocada entre as sensações corporais naturais, provenientes do mecanismo biológico de "luta e fuga" (Barlow, 1988) com a possibilidade de essas sensações serem percebidas como prenúncio de uma doença grave ou morte iminente, origina o TP. Sendo assim, por associação, sempre que surgirem no indivíduo, sensações corporais provenientes de exercícios físicos (Araújo, Mello, \& Leite, 2006) ou adaptações fisiológicas, automaticamente serão entendidas e experimentadas como sensações desconfortáveis precursoras de alguma situa- 
ção grave. Esse modelo de padrão indevido de funcionamento que interpreta as sensações físicas normais (Clark et al., 1988) como originárias de algo temido de maior conseqüência, leva o indivíduo a evitar, muitas vezes, exercícios físicos, estímulos sexuais, alterações térmicas, entre outras, por associarem as sensações corporais inofensivas, decorrentes dessas práticas, com aquelas similares sentidas durante os ataques de pânico (Ehlers \& Margraf,1989). As sensações corporais afins são: falta de ar, suores, tonturas, taquicardia, entre outras.

Os ataques de pânico (Lang \& Craske, 2003) passam a ser cíclicos em conseqüência de ter se estabelecido uma cognição catastrófica que se tornou o padrão de comportamento do indivíduo.

As anormalidades respiratórias estão comumente associadas com a ansiedade em geral e em especial com os ataques de pânico (Valença et al., 1996). A função respiratória (King, Valença, Melo-Neto, \& Nardi, 2007) desempenha um fator importante nos pacientes com TP, devido a freqüente presença de sintomas relacionados com alterações da respiração. Observamos diferenças no comportamento dos sintomas respiratórios entre indivíduos com TP. Briggs, Stretch e Brandon (1993) procuraram definir as diferenças, classificando o TP em subtipo respiratório (SR) e subtipo não-respiratório (SNR). Os pacientes do SR, diferentes do SNR, apresentam predomínio de sintomas como sensação de sufocação, dificuldade de respirar, parestesias, tontura e medo de morrer.

Outro sintoma importante nestes pacientes é a hiperventilação (King, Valença, \& Nardi, 2008). Esta, quando acontece durante um período prolongado, leva o indivíduo a um estado de hipocapnia, ou seja, a uma alcalose respiratória crônica que se manifesta por dispnéia e taquicardia. Pesquisadores (Papp, Klein, \& Gorman, 1993) observaram, em alguns pacientes, baixos níveis de $\mathrm{CO}^{2}$, em função da hiperventilação crônica, e que os ataques de pânico estariam relacionados a uma hipersensibilidade do sistema nervoso autônomo ao dióxido de carbono.

A sensação de esforço respiratório é experimentada de modo aversivo pelo sujeito. Estudos demonstram (Biber \& Alkin, 1999) que o TP do SR é induzido por uma maior sensibilidade ao acúmulo de $\mathrm{CO}^{2}$ no organismo, desencadeando crises de pânico.

A terapia cognitivo-comportamental (TCC; Hawton, Salkovskis, Kirk, \& Clark, 1997) abrange técnicas específicas direcionadas a reorganizar e/ou neutralizar comportamentos desadaptativos provenientes do TP, com a vantagem de ser uma terapia breve. Dentre essas técnicas, estão os exercícios respiratórios e os exercícios de indução dos sintomas (King et al., 2008), fundamentais no tratamento do SR, que facilitam aos pacientes a percepção dos descontroles da respiração e de como lidar com essas alterações. Uma vez entendido o processo respiratório e retomado o equilíbrio da respiração, o indivíduo, por intermédio de reestruturações cognitivas, começaria a perceber que os sintomas emer- gentes teriam causa própria, seriam oriundos do mecanismo fisiológico de adaptação, e que não teriam a gravidade e as conseqüências temidas e que eram esperadas anteriormente.

Este estudo propõe 8 sessões de TCC, individuais e semanais com uma hora de duração. Além de os pacientes serem ouvidos em suas questões e sintomas, as sessões de TCC, apresentam também, um conteúdo didático padrão para todas as sessões, para que o paciente entenda a origem, natureza e trajetória do TP.

O objetivo do estudo foi verificar a resposta dos pacientes do SR e SNR às técnicas de TCC. A hipótese é que o grupo "Intervenção" que recebeu a associação das intervenções de medicação e TCC apresentaria melhora significativa nos sintomas respiratórios relacionados aos ataques de pânico, comparados a um grupo "Controle" que recebeu apenas medicação sem TCC, no mesmo período de tratamento.

\section{Método}

O estudo descritivo e quantitativo compreendeu uma amostra randomizada de 50 pacientes voluntários com o diagnóstico de TP com agorafobia, atendidos no Laboratório de Pânico e Respiração do Instituto de Psiquiatria da Universidade Federal do Rio de Janeiro (LABPR/ IPUB/UFRJ). Médicos psiquiatras realizaram o diagnóstico de acordo com o DSM-IV-TR (APA, 2000), com aplicação da Structured Clinical Interview Diagnostic (SCID-I; First, Spitzer, Gibbon, \& Williams, 1997). A prescrição médica consistiu em antidepressivos tricíclicos ou inibidores seletivos de recaptação da serotonina.

Os pacientes que foram incluídos no estudo eram maiores de 18 anos, de ambos os sexos e sem comorbidades graves. Pacientes dependentes de álcool e/ou drogas, retardo mental ou transtornos mentais graves foram excluídos do estudo.

Todos os voluntários que concordaram em participar do estudo foram informados dos procedimentos da pesquisa e assinaram um "Termo de Consentimento Livre e Esclarecido" de acordo com o código de ética em pesquisa em seres humanos. O projeto foi aprovado pelo Comitê de Ética em Pesquisa (CEP - IPUB/UFRJ), seguindo a orientação da Declaração de Helsinque.

A amostra foi dividida, por meio de sorteio, em dois grupos. O primeiro, denominado grupo União, foi composto por 25 indivíduos que receberam 8 sessões de TCC com uso de medicação. O segundo, chamado grupo Controle, também com 25 indivíduos, recebeu apenas medicação sem TCC.

Com a finalidade de comparar os resultados dos grupos após as intervenções, foram aplicados no início e ao final da pesquisa instrumentos de avaliação, como: inventário de ansiedade de Beck (BIA; Beck \& Emery, 1985); faixa do inventário de ansiedade de Beck (FBIA) com os escores Mínimo $(0-10)$, Leve $(11$ - 19), Mode- 
rado (20 a 30) e Grave (31 - 63); escala de incapacidade de Sheehan (Sheehan, 1983; total, trabalho, social e família [EISheeham]); escala de problemas psicossociais e ambientais ([PPA, EIXO IV], APA, 2000); escala de avaliação global do funcionamento (0 a 100; [AGF, EIXO V], APA, 2000); questionário de medos e fobias (QMF; Marks \& Matheus, 1979); escala de cognições agorafóbicas (ECA; Chambless, Caputo, Bright, \& Gallagher, 1984); questionário de sensações corporais (QSC; Chambless et al., 1984) e escala para pânico e agorafobia (EPA; Bandelow, 1999). Esse estudo adota como critério de significância $p<0,05$.

Quanto aos parâmetros psicométricos, a BIA apresenta alfa de 0,92 e 0,91 para população de pacientes diagnosticados com pânico sem e com agorafobia, respectivamente. No estudo brasileiro (Cunha, 2001) teve sua validade de conteúdo estudada com base na comparação com o IDATE (Spielberger, Gorsuch, \& Lushene, 1979), apresentando relação significativa com Estado $(r=0,76$; $p<0,001)$ e Traço $(r=0,78 ; p<0,001)$. Os valores obtidos para correlação entre BDI e BAI em amostra psiquiátrica para população de pânico sem e com agorafobia foram, respectivamente, $r=0,37$ e $r=0,50$ considerando $p<0,001$.

As demais escalas não foram adaptadas para a população brasileira, sendo utilizadas apenas em pesquisas, sem possuir normatização que permita comparação externa ao grupo pesquisado. Para esse estudo os dados obtidos com esses instrumentos serviram para a comparação dos resultados dentre e entre grupos.

O conteúdo das oito sessões de TCC foi distribuído da seguinte forma: educação sobre a trajetória do TP com os conceitos didáticos de ansiedade, agorafobia, pânico, hiperventilação, exercícios de indução dos sintomas, exercícios de reeducação da respiração e exercícios de relaxamento muscular. Todas as questões dos pacientes foram ouvidas. Procuramos identificar e elaborar com reestruturação cognitiva às cognições mal-adaptativas.

\section{Modelo Padrão das 8 Sessões de TCC}

$1^{a}$. Sessão. Educação didática sobre a trajetória do TP: psicoeducação com os conceitos de ansiedade, medo, respiração, hiperventilação, pânico e agorafobia.

$2^{a}$. Sessão. O mecanismo fisiológico de "luta e fuga", semelhança entre as sensações provenientes desse mecanismo e das sensações corporais emergentes do pânico. Os exercícios de reeducação da respiração usados em todas as sessões: Inspirar lentamente pelo nariz contando até três, prender a respiração contando até três, exalar lentamente o ar pela boca contando até seis. Repetir algumas vezes seguidas a respiração.

$3^{a}$. Sessão. O modelo de hiperventilação se define como um ritmo e uma profundidade de respiração exagerada para as necessidades do corpo em um momento específico. Realizamos com o paciente sentado um exercício de indução dos sintomas de hiperventilação: Inspirar e expirar profundamente por 90 segundos, em seguida se le- vantar e olhar fixo um ponto de luz no teto. Realizamos o exercício de reeducação da respiração e o de relaxamento muscular abrangendo quatro grupamentos musculares do corpo. São eles: $1^{\circ}$ ombros, braços e mãos, $2^{\circ}$ abdômen e peitoral, $3^{\circ}$ rosto e pescoço, $4^{\circ}$ glúteos, genitais, pernas e pés. Contrair cada grupamento de músculos durante 10 segundos ao mesmo tempo inspirando pelo nariz e em seguida relaxar a musculatura expirando pela boca.

$4^{a}$. Sessão. Um exercício de indução dos sintomas: O paciente de pé vira a cabeça de um lado para o outro durante 30 segundos, pára e tenta fixar os olhos em um ponto na parede. Em seguida, avaliamos as sensações corporais resultantes e procuramos entender a causa. Repetimos o exercício de indução dos sintomas da $3^{\mathrm{a}}$. Sessão. Recuperamos o equilíbrio físico e respiratório do paciente com o exercício de reeducação da respiração. Com isso, o paciente entende a origem dos sintomas e percebe que são inofensivos.

$5^{a}$. Sessão. Conceitos sobre as sensações corporais que podem ser acentuadas devido à situação ou substâncias. Exemplo: exercita-se e mexer-se rapidamente, mudança de temperatura, luzes brilhantes, cafeína, álcool, medicamentos e padrões irregulares de respiração. O pânico tem suas raízes no medo das sensações físicas. Realizamos com o paciente um exercício de indução dos sintomas, o de girar no lugar por um minuto inspirando e expirando profundamente, depois, parar e compreender as reações decorrentes e resignificá-las. Em seguida, o exercício de reeducação da respiração e o de relaxamento muscular.

$6^{a}$. Sessão. No TP algumas queixas são: Ex: "não consigo respirar", ou "vou sufocar". Explicamos que é natural respirar excessivamente quando se está ansioso. O corpo busca energia na forma de oxigênio para se preparar para lidar com o perigo. Quando o oxigênio não é usado na mesma proporção em que é consumido, resulta em hiperventilação. Relembramos os conceitos da hiperventilação e repetimos os exercícios de indução dos sintomas da $4^{\mathrm{a}}$. Sessão, em seguida o exercício de reeducação da respiração e o de relaxamento muscular.

$7^{a}$. Sessão. Educação sobre Reestruturação Cognitiva: é fundamental o paciente compreender que o modo como os eventos são interpretados é que determinam à natureza das reações emocionais resultantes. Nesta sessão, procuramos ajudar o paciente a identificar as cognições mal-adaptativas que o estão levando às respostas sintomáticas, buscamos reencontrar as causas originais e desfazer as interpretações distorcidas.

8. Sessão. Exposição Interoceptiva (King et al., 2007): refere-se ao medo aprendido de estados internos. Certas sensações de terror, similares a medos antes experimentados, costumam sinalizar possíveis novos ataques de pânico. O estímulo automático gerado intensifica as sensações temidas criando um círculo vicioso. Realizamos dois exercícios de indução dos sintomas e analisamos os 
sintomas que originaram destes exercícios procurando entender as causas. Exercícios de indução dos sintomas: $\mathrm{N}^{\circ} 1$ - Sentar, colocar a cabeça entre as pernas durante 30 segundos, depois levantar rapidamente e olhar para o teto. $\mathrm{E} \mathrm{N}^{\circ} 2$ - segurar a respiração até não agüentar mais girando em torno de si mesmo (30 segundos). Em seguida realizamos o exercício de reeducação da respiração.

Após o término de todos os procedimentos da pesquisa, dentro do mesmo espaço de tempo, reavaliamos os dois grupos com os mesmos instrumentos iniciais. Comparamos os grupos, observamos as mudanças ocorridas, os benefícios, prejuízos e diferenças ocasionadas por um grupo estar recebendo acompanhamento com TCC e medicação comparado com outro controle que apenas medicação sem TCC.

\section{Resultados}

O grupo que realizou 8 sessões de TCC com medicação foi chamado de grupo de Intervenção e o grupo com medicação sem TCC de Controle.

O grupo controle apresenta a seguinte formação: total de 25 sujeitos; $21 \%$ femininos e $4 \%$ masculinos. Quanto à escolaridade: $8 \%$ primeiro grau, $11 \%$ segundo grau, $6 \%$ nível superior. Quanto ao estado civil: $9 \%$ solteiros, $10 \%$ casados e $6 \%$ separados. Religião: $12 \%$ católicos, $8 \%$ protestantes ou evangélicos, $1 \%$ espírita e $4 \%$ outras formas de religião. Comorbidades clínicas: $60 \%$ sim, $40 \%$ não. Comorbidades psíquicas: $68 \%$ sim e $32 \%$ não. Tabagismo: $14 \%$ não fumantes, $9 \%$ ex-fumantes e $2 \%$ fumantes. Etnia auto-referida: $76 \%$ brancos, $20 \%$ pardos,
4\% negros. Ocupação: 36\% estudantes ou "do lar", $64 \%$ empregados no momento. Os participantes desse grupo apresentam ainda média de 33,68 para idade, 10,80 para anos de estudo, $\mathrm{R} \$ 519,20$ como renda individual média e $\mathrm{R} \$ 1468,48$ como renda familiar média. Presença do subtipo respiratório: $76 \%$.

O grupo de intervenção apresenta a seguinte formação: total de 25 sujeitos; $18 \%$ femininos e $7 \%$ masculinos. Quanto à escolaridade: 6\% primeiro grau, $8 \%$ segundo grau, $11 \%$ nível superior. Quanto ao estado civil: $9 \%$ solteiros, $11 \%$ casados e $5 \%$ separados. Religião: $19 \%$ católicos, $3 \%$ protestantes ou evangélicos, $2 \%$ espírita e $1 \%$ outras formas de religião. Comorbidades clínicas: $56 \%$ sim, $44 \%$ não. Comorbidades psíquicas: $28 \%$ sim e $72 \%$ não. Tabagismo: $8 \%$ não fumantes, $12 \%$ ex-fumantes e $5 \%$ fumantes. Etnia auto-referida: $80 \%$ brancos, $16 \%$ pardos, $4 \%$ negros. Ocupação: $16 \%$ estudantes ou "do lar", $44 \%$ empregados no momento, $40 \%$ estão em afastamento do trabalho devido a licença. Os participantes desse grupo apresentam ainda média de 44,48 para idade, 12,84 para anos de estudo, $\mathrm{R} \$ 1154,52$ como renda individual média e R\$2481,67 como renda familiar média. Presença do subtipo respiratório: $68 \%$.

\section{Análise dos Dados}

A Análise do Subtipo Respiratório do Grupo Intervenção com avaliação ao Início e Término do período de tratamento é apresentada na Tabela 1 , sendo a mesma análise, para o subtipo Não Respiratório demonstrada na Tabela 2. Em ambas as tabelas são descritos apenas as avaliações que atingiram o nível de significância adotado para esse estudo.

Tabela 1

Análise do Subtipo Respiratório do Grupo Intervenção com Avaliação ao Início e Término do Período de Tratamento

\begin{tabular}{lrcc}
\hline Teste & Início & Término & ANOVA \\
\hline BIA & 35,9 & 21,3 & 0,007 \\
EISheeham - Total & 15,6 & 4,6 & 0,001 \\
EISheeham - Trabalho & 5,6 & 0,5 & 0,000 \\
EISheeham - Social & 4,9 & 2,3 & 0,037 \\
EISheeham - Familia & 5,1 & 1,8 & 0,004 \\
FBIA & 3,4 & 2,5 & 0,016 \\
ECA Total & 2,9 & 2,2 & 0,021 \\
ECA - Perda de controle & 3,0 & 2,1 & 0,024 \\
QSC & 3,2 & 2,4 & 0,007 \\
EPA - Total & 30,3 & 18,3 & 0,015 \\
EPA - Ataque de Pânico & 1,6 & 0,7 & 0,009 \\
EPA - Ansiedade Antecipatória & 3,0 & 2,0 & 0,030 \\
EPA - Preocupações com a saúde & 2,3 & 1,4 & 0,021 \\
EAGF & 55,8 & 70,9 & 0,007
\end{tabular}

Nota. Siglas: Beck inventário de ansiedade (BIA); Faixa do Beck inventário de ansiedade (FBIA); Escala de incapacidade de Sheehan (EISheehan); Escala de cognições agorafóbicas (ECA); Questionário de sensações corporais (QSC); Escala para pânico e agorafobia (EPA); Escala de avaliação global do funcionamento (EAGF). 
King, A. L. S., Valença, A. M., Simões Neto, J. P., Nardi, A. E. \& Silva, A. C. O. (2012). Subtipo Respiratório Versus Não Respiratório no Transtorno de Pânico com Agorafobia: Avaliação com Terapia Cognitivo-Comportamental.

Tabela 2

Análise do Subtipo Não Respiratório do Grupo Intervenção com Avaliação ao Início e Término do Perído de Tratamento

\begin{tabular}{lccc}
\hline Teste & Início & Término & ANOVA \\
\hline BIA & 36,3 & 17,7 & 0,038 \\
EISheeham - Trabalho & 4,0 & 0,3 & 0,038 \\
FBIA & 3,8 & 2,0 & 0,010 \\
\hline
\end{tabular}

Nota. Siglas: Beck inventário de ansiedade (BIA); Faixa do Beck inventário de ansiedade (FBIA); Escala de incapacidade de Sheehan (EISheehan).

No Grupo Controle não foram observadas mudanças significativas entre o início e o fim do período determinado para tratamento, tanto no Subtipo Respiratório quanto no Subtipo Não Respiratório.

Na comparação entre os pacientes do Grupo Intervenção e Grupo Controle no final do tratamento (Tabela 3), pode-se perceber que só houve resultados positivos no SR. O SNR não apresentou nenhum resultado significa- tivo, por isso, não aparece nas tabelas. Assim como na análise geral, houve uma diminuição de resultados significativos quando se compara Intervenção-Início $\mathrm{x}$ Intervenção-Fim no SR [8 resultados significativos (Tabela 1), versus Intervenção-Fim x Controle-Fim no SR (Tabela 3)] resultados significativos. No entanto, pode-se ressaltar que, com esta diminuição, não foi registrado nenhum resultado significativo no SNR, concentrando-se todos os resultados significativos no SR.

Tabela 3

Análise Comparativa dos Resultados do Subtipo Respiratório do Grupo Intervenção X Grupo Controle ao Término do Período de Tratamento

\begin{tabular}{lccc}
\hline \multicolumn{1}{c}{ Teste } & Intervenção & Controle & ANOVA \\
\hline EISheehan - Total & 4,6 & 10,7 & 0,023 \\
EISheehan - Trabalho & 0,5 & 3,0 & 0,007 \\
EISheehan - Familia & 1,8 & 4,1 & 0,033 \\
Ataques de pânico/Precauções & 1,4 & 2,3 & 0,024 \\
\hline
\end{tabular}

Nota. Siglas: Escala de incapacidade de Sheehan (EISheehan).

Tabela 4

Análise Comparativa dos Resultados do Subtipo Respiratório do Grupo Intervenção X Grupo Controle ao quanto ao Acesso a Cuidados de Saúde

\begin{tabular}{lrrrrrr}
\hline \multirow{2}{*}{ EPPA - Acesso a cuidados à Saúde } & \multicolumn{3}{c}{ Intervenção } & \multicolumn{3}{c}{ Controle } \\
\cline { 2 - 7 } & Início & \multicolumn{1}{c}{ Fim } & Total & Início & Fim & Total \\
\hline Sim & 25,0 & 0,0 & 11,8 & 0,0 & 20,0 & 10,5 \\
Não & 75,0 & 100,0 & 88,2 & 100,0 & 80,0 & 89,5 \\
Total & 100,0 & 100,0 & 100,0 & 100,0 & 100,0 & 100,0 \\
Qui-quadrado & & 0,024 & & & 0,045 & \\
\hline
\end{tabular}

Nota. Escala de problemas psicossociais e ambientais (EPPA).

$\mathrm{Na}$ análise geral, os resultados dos testes entre pacientes do grupo de Intervenção no início e no final do tratamento apresentaram muitas diferenças significativas. Entretanto, os resultados foram mais expressivos no SR do que no SNR. No SR, 8 testes apresentaram diferenças significativas no BIA (redução da ansiedade, da sensação de sufocação, da dificuldade de respirar), FBIA (redução da ansiedade de moderada para leve), EISheehan (redução significativa do sentimento de incapacidade geral, no trabalho, na vida social e familiar), ECA (redução dos escores total que significa redução do medo de perder o controle e do medo de ter problemas físicos), QSC (redução da falta de ar, do coração disparado, da tontura), EPA (redução dos AP, do comportamento de esquiva), AGF (escore de 0 a 100) (aumento de 55,8 para 70,9 significando um aumento do bem-estar global) e PPA 
(redução nos problemas sociais e ocupacionais). No SNR, as diferenças só foram percebidas em 3 testes, sendo: BIA (redução da ansiedade em geral), FBIA (redução da ansiedade de moderada para leve) e EISheehan-trabalho (aumento da capacidade para o trabalho).

Os pacientes do SR aumentaram seu bem estar, segundo a escala de avaliação global do funcionamento de 55,8 para 70,9 em comparação com o SNR.

\section{Discussão}

A classificação do TP em subtipos pode ser útil para elucidação fisiopatológica e resposta farmacológica deste transtorno. A pesquisa em questão revelou $70 \%$ de pacientes com SR. Acreditamos que os resultados poderiam ter sido mais precisos se tivéssemos determinado uma medicação específica para cada subtipo de TP.

Briggs et al. (1993) demonstraram uma resposta de tratamento favorável com imipramina em pacientes do SR comparados com pacientes do SNR. Uma visão geral (Holt \& Andrews, 1989) sobre a década passada diz respeito ao uso de clonazepan em uma variedade de transtornos psiquiátricos. A eficácia do clonazepan para o tratamento em curto prazo do TP foi estabelecida inteiramente no final dos anos 90.

Alguns autores (Valença, Nardi, Nascimento, Mezzasalma, \& Zin, 2000) procuraram verificar a sensibilidade de pacientes com TP ao teste de indução de ataques de pânico com dióxido de carbono a $35 \%$ e analisar a intensidade, a duração e a sintomatologia dos ataques de pânico produzidos por esse agente em laboratório, comparando-os com os ataques de pânico espontâneos nestes pacientes.

Os mecanismos que produzem ansiedade e pânico por inalação do dióxido de carbono não é compreendida inteiramente (Bailey, Argyropoulos, Lightman, \& Nutt, 2003), embora mais recentemente haja uma evidência para sugerir a participação de um "circuito neural do medo". Sugerem que este circuito neural do medo esteja associado em parte pela rede da noradrenalina no cérebro.

Evidências crescentes na literatura (Lopes, Nardi, Nascimento, Valença, \& Zin, 2002) vêm revelando a conexão pânico-respiração em diversas situações. Neste estudo os autores relatam o caso de três pacientes com TP com ataques de pânico no sono com sintomas respiratórios proeminentes.

Muotri, Nunes e Bernik (2007) realizaram um estudo sobre TP e esquiva de atividade física e sua possível associação com um subtipo descrito de TP, o SR. Como os pacientes tendem a interpretar as mudanças autonômicas ocasionadas pelo exercício físico como um gatilho para o ataque de pânico, é possível que estes pacientes desenvolvessem esquiva de atividade física.

King et al. (2008) relataram três casos graves de TP do SR, com agorafobia, cujos sintomas de hiperventilação predominavam sobre os demais. $\mathrm{O}$ tratamento consistiu na prescrição de antidepressivos tricíclicos e no encaminha- mento para sessões de TCC. Demonstraram a evolução satisfatória do tratamento com a técnica dos exercícios de indução dos sintomas em ambiente de laboratório para a redução e/ou extinção dos sintomas hiperventilatórios, entre outros. Esse procedimento foi considerado fundamental na evolução do tratamento e permitiu preparar os doentes para enfrentarem as situações agorafóbicas subsequentes.

Com metodologia semelhante (King et al., 2007), outros autores observaram que pacientes com o diagnóstico de TP com agorafobia do SR apresentaram boa resposta à TCC e aos exercícios de indução dos sintomas, após correção do padrão inadequado de respiração (torácica), seguidos de reestruturação de cognições distorcidas.

Estudos (Wilhelm, Gerlach, \& Roth, 2001) têm demonstrado que $50 \%$ e $60 \%$ dos pacientes descrevem sintomas de hiperventilação como sendo muito semelhante aos sintomas de ataques de pânico. Os pacientes relatavam sintomas similares aos do pânico, principalmente relacionados ao descontrole da freqüência respiratória.

Em artigo de revisão (Araújo et al., 2006), abordaram a possibilidade do uso do exercício físico como forma de terapia de exposição a estímulos interoceptivos no tratamento do TP, especificamente o do SR. Concluíram que a exposição é o fator essencial do tratamento e que se podem tratar os ataques de pânico pela exposição a sensações físicas experimentadas durante estes ataques. Consideram que o modo mais fácil de provocar essas sensações é através da hiperventilação.

\section{Conclusão}

O aprendizado das 8 sessões de TCC, de que as sensações corporais de pânico não eram realmente perigosas foi primordial para os indivíduos superarem a agorafobia e controlarem o pânico. Quando os pacientes conseguiram alterar por intermédio das técnicas da reestruturação cognitiva os pensamentos referentes à capacidade de lidar com o controle da respiração e com as situações temidas, conseguiram também, controlar os sintomas físicos.

Quanto à limitação do estudo, seria importante uma definição específica da medicação para cada grupo do estudo para que obtivéssemos resultados mais precisos.

Consideramos fundamentais para os resultados satisfatórios do tratamento algumas técnicas da TCC como os exercícios de indução dos sintomas e os de reeducação da respiração, principalmente para a compreensão didática do TP e da hiperventilação. Percebendo, por intermédio de reestruturação cognitiva que os sintomas respiratórios emergentes eram inofensivos, os pacientes foram capazes de perceber que podiam lidar com eles, diminuindo assim, a ansiedade e os ataques de pânico.

Segundo os próprios pacientes do estudo, foram possíveis a recuperação da autoconfiança e consequentemente a autoestima. Estes conseguiram reduzir as preocupações, expandir seus horizontes anteriormente limitados, principalmente por lugares fechados ou situações que de- 
sencadeassem falta de ar, dificuldade em respirar e sensação de sufocamento.

Verificamos neste estudo que, tanto no grupo Intervenção quanto no grupo Controle, os pacientes do SR apresentaram um resultado mais positivo no tratamento em geral do que os pacientes do SNR.

\section{Referências}

Araújo, S. R. C., Mello, M. T., \& Leite, J. R. (2006). Transtorno de ansiedade e exercícios físicos. Revista Brasileira de Psiquiatria, 29, 164-171.

Associação Americana de Psiquiatria. (2000). DSM-IV-TR, Manual diagnóstico e estatístico de transtornos mentais (5. ed.). Porto Alegre, RS: Artes Médicas.

Bailey, J. E., Argyropoulos, S. V., Lightman, S. L., \& Nutt, D. J. (2003). Does the brain noradrenaline network mediate the effects of the $\mathrm{CO}^{2}$ challenge? Journal of Psychopharmacology, 17, 252-259.

Bandelow, B. (1999). Panic and Agoraphobia Scale (PAS). Seattle, WA: Hogrefe \& Huber.

Barlow, D. H. (1988). Anxiety and its disorders: The treatment of anxiety and panic. New York: Guilford Press.

Beck, A. T., \& Emery, G. (1985). Anxiety disorders and phobias: A cognitive perspective. New York: Basic Books.

Biber, B., \& Alkin, T. (1999). Panic disorders subtypes: Differential responses to $\mathrm{CO}^{2}$ challenge. American Journal of Psychiatry, 156, 739-744.

Briggs, A. C., Stretch, D. D., \& Brandon, S. (1993). Subtyping of the panic disorder by symptom profile. British Journal of Psychiatry, 163, 201-209.

Chambless, D. L., Caputo, G. C., Bright, P., \& Gallagher, R. (1984). Assessment of "fear of fear" in agoraphobics: The Body Sensations Questionnaire and the Agoraphobic Cognitions Questionnaire. Journal of Consulting and Clinical Psychology, 52, 1090-1097.

Clark, D. M., Salkovskis, P., Gelder, M., Koehler, C., Martin, M., \& Anastasiades, P. (1988). Tests of a cognitive theory of panic. In I. Hand \& H. Wittchen (Eds.), Panic and phobias: Treatments and variables affecting course and outcome (Vol. 2, pp. 71-90). Berlin, Germany: Springer-Verlag.

Cunha, J. A. (2001) Manual da versão em português das Escalas Beck. São Paulo, SP: Casa do Psicólogo.

Ehlers, A., \& Margraf, P.(1989). The psycho physiological modelo of panic attacks. In P. M. G. Emmelkamp, W. T. Everaed, F. W. Kraaimaat, \& M. J. Van Son (Eds.), Fresh perspectives on anxiety disorders (pp. 1-29). Lisse, Netherlands: Sweets \& Zeitlinger.

First, M. B., Spitzer, R. L., Gibbon, M., \& Williams, J. B. M. (1997). Structured Clinical Interview Diagnostic (SCID) for DSM-IV Axis I Disorders-Clinician Version (SCID-CV). Washington, DC: American Psychiatric Press.

Hawton, K., Salkovskis, P. M., Kirk, J., \& Clark, D. M. (1997). Terapia cognitivo-comportamental para problemas psiquiátricos, um guia prático. São Paulo, SP: Martins Fontes.

Holt, P., \& Andrews, G. (1989). Hyperventilation and anxiety in panic disorder, agoraphobia, and generalized anxiety disorder. Behavior Research and Therapy, 27, 453-460.

King, A. L. S., Valença, A. M., Melo-Neto, V. L., \& Nardi, A. E. (2007). A importância do foco da terapia cognitivocomportamental direcionada às sensações corporais no transtorno de pânico: Relato de caso. Revista de Psiquiatria Clínica, 34, 191-195.
King, A. L. S., Valença, A. M., \& Nardi, A. E. (2008). Hiperventilação. A terapia cognitivo-comportamental e a técnica dos exercícios de indução dos sintomas no transtorno de pânico. Revista Portuguesa de Pneumologia, 14, 303-308.

Lang, A. J., \& Craske, M. G. (2003). Pânico e fobia. In J. R. White \& A. S. Freeman (Eds.), Terapia cognitivocomportamental em grupo para populações e problemas especificos (pp. 71-107). São Paulo, SP: Roca.

Lopes, F. L., Nardi, A. E., Nascimento, I., Valença, A. M., \& Zin, W. A. (2002). Nocturnal panic attacks. Arquivos de Neuro-Psiquiatria, 60, 717-720.

Marks, I. M., \& Mathews, A. M. (1979). Brief standard selfrating for phobic patients. Fear and Phobia Questionnaire. Behavior Research Therapy, 17, 263-267.

Muotri, R. W., Nunes, R. P., \& Bernik, M. A (2007). Exercícios aeróbio como terapia de exposição a estímulos interoceptivos no tratamento do transtorno de pânico. Revista Brasileira de Medicina do Esporte, 13, 327-330.

Papp, L. A., Klein, D. F., \& Gorman, J. M. (1993). Carbon dioxide hypersensitivity, hyperventilation, and panic disorder. American Journal of Psychiatry, 150(8), 1149-1157.

Sheehan, D. V. (1983). The anxiety disease - Sheehan Disability Scale. New York: Charles Scribner's Sons.

Spielberger, C. D., Gorsuch, R. L., \& Lushene, R. E. (1979). Inventário de Ansiedade Traço-Estado: Manual. Rio de Janeiro, RJ: Centro Editor Psicologia Aplicada.

Valença, A. M., Nardi, A. E., Kinrys, G., Andrade, Y., Figueira, I., Mendlowicz, M., et al. (1996). Transtorno de pânico e respiração. Jornal Brasileiro de Psiquiatria, 45, 491-496.

Valença, A. M., Nardi, A. E., Nascimento, I., Mezzasalma, M. A., \& Zin, W. (2000). Ataques de pânico provocado pelo dióxido de carbono: Etiopatogenia, bloqueio através de psicofármacos, e terapia cognitivo-comportamental. Jornal Brasileiro de Psiquiatria, 49, 69-80.

Wilhelm, F. H., Gerlach, A. L., \& Roth, W. T. (2001). Slow recovery from voluntary hyperventilation in panic disorder. Psychosomatic Medicine, 63, 638-649.
Recebido: 02/12/2009

$1^{a}$ revisão: $25 / 11 / 2010$ Aceite final: $29 / 11 / 2010$ 